Edith Cowan University

Research Online

Research outputs 2012

$1-1-2012$

Predictors of rapid cognitive decline in Alzheimer's disease:

Results from the Australian Imaging, Biomarkers and Lifestyle

(AIBL) study of ageing

\author{
A Sona \\ P Zhang \\ D Ames \\ A I Bush \\ N T Lautenschlager
}

See next page for additional authors

Follow this and additional works at: https://ro.ecu.edu.au/ecuworks2012

Part of the Medicine and Health Sciences Commons

10.1017/S1041610211001335

This is an Author's Accepted Manuscript of: Sona, A., Zhang, P., Ames, D., Bush, A., Lautenschlager, N., Martins, R. N., Masters, C., Rowe, C., Szoeke, C., Taddei, K. , \& Ellis, K. (2012). Predictors of rapid cognitive decline in Alzheimer's disease: Results from the Australian Imaging, Biomarkers and Lifestyle (AIBL) study of ageing. International Psychogeriatrics, 24(2), 197-204. Available here

This version is free to view and download for private research and study only. Not for re-distribution, re-sale or use in derivative works. (c) International Psychogeriatrics.

This Journal Article is posted at Research Online.

https://ro.ecu.edu.au/ecuworks2012/314 


\section{Authors}

A Sona, P Zhang, D Ames, A I Bush, N T Lautenschlager, Ralph Martins, C L Masters, C C Rowe, C Szoeke, Kevin Taddei, and Kathryn A Ellis

This journal article is available at Research Online: https://ro.ecu.edu.au/ecuworks2012/314 


\title{
Predictors of rapid cognitive decline in Alzheimer's disease: results from the Australian Imaging, Biomarkers and Lifestyle (AIBL) study of ageing
}

\author{
Alessandro Sona ${ }^{1,3}$ Ping Zhang, ${ }^{2}$ David Ames, ${ }^{3,4}$ Ashley I. Bush, ${ }^{5}$ Nicola T. \\ Lautenschlager, ${ }^{4,6}$ Ralph N. Martins, 7,8 Colin L. Masters, ${ }^{5,9}$ Christopher C. Rowe, ${ }^{10}$ \\ Cassandra Szoeke, ${ }^{2,3}$ Kevin Taddei, ${ }^{7,8}$ Kathryn A. Ellis ${ }^{4,5}$ and AIBL Research Group ${ }^{11}$ \\ ${ }^{1}$ AOU San Giovanni Battista - Molinette, Geriatria e Malattie Metaboliche dell'Osso, Università degli Studi di Torino, Torino, Italy \\ ${ }^{2}$ Commonwealth Scientific and Industrial Research Organisation (CSIRO), Parkville, Victoria, Australia \\ ${ }^{3}$ National Ageing Research Institute, Parkville, Victoria, Australia \\ ${ }^{4}$ Academic Unit for Psychiatry of Old Age, University of Melbourne Department of Psychiatry, St. Vincent's Aged Psychiatry Service, St George's Hospital, \\ Kew, Victoria, Australia \\ ${ }^{5}$ Mental Health Research Institute, Parkville, Victoria, Australia \\ ${ }^{6}$ Centre of Excellence for Alzheimer's Disease Research \& Care, School of Psychiatry and Clinical Neurosciences and WA Centre for Health and Ageing, \\ University of Western Australia, Perth, Western Australia, Australia \\ ${ }^{7}$ School of Exercise, Biomedical and Health Sciences, Edith Cowan University, foondalup, Western Australia, Australia \\ ${ }^{8}$ Sir Fames McCusker Alzheimer's Disease Research Unit (Hollywood Private Hospital), Perth, WA, Australia; \\ ${ }^{9}$ University of Melbourne, Parkville, Vic, Australia; \\ ${ }^{10}$ Austin PET Centre, and University of Melbourne Department of Medicine, Austin Health, Heidelberg, Victoria, Australia \\ ${ }^{11}$ For a full list of the AIBL Research Group see www.aibl.csiro.au
}

Background: The AIBL study, which commenced in November 2006, is a two-center prospective study of a cohort of 1112 volunteers aged 60+. The cohort includes 211 patients meeting NINCDS-ADRDA criteria for Alzheimer's disease (AD) (180 probable and 31 possible). We aimed to identify factors associated with rapid cognitive decline over 18 months in this cohort of $\mathrm{AD}$ patients.

Methods: We defined rapid cognitive decline as a drop of 6 points or more on the Mini-Mental State Examination (MMSE) between baseline and 18-month follow-up. Analyses were also conducted with a threshold of 4, 5, 7 and 8 points, as well as with and without subjects who had died or were too severely affected to be interviewed at 18 months and after, both including and excluding subjects whose AD diagnosis was "possible" AD. We sought correlations between rapid cognitive decline and demographic, clinical and biological variables.

Results: Of the $211 \mathrm{AD}$ patients recruited at baseline, we had available data for $156(73.9 \%)$ patients at 18 months. Fifty-one patients were considered rapid cognitive decliners $(32.7 \%)$. A higher Clinical Dementia Rating scale (CDR) and higher CDR "sum of boxes" score at baseline were the major predictors of rapid cognitive decline in this population. Furthermore, using logistic regression model analysis, patients treated with a cholinesterase inhibitor (CheI) had a higher risk of being rapid cognitive decliners, as did males and those of younger age.

Conclusions: Almost one third of patients satisfying established research criteria for $\mathrm{AD}$ experienced rapid cognitive decline. Worse baseline functional and cognitive status and treatment with a CheI were the major factors associated with rapid cognitive decline over 18 months in this population.

Key words: dementia, cognitive testing, memory, cognitive disorders, cholinesterase inhibitor

\section{Introduction}

In Australia the percentage of the total population with dementia is expected to rise from $1 \%$ at

Correspondence should be addressed to: Professor David Ames, Director, National Ageing Research Institute, P.O. Box 2127, Royal Melbourne Hospital, Victoria 3050, Australia. Phone: +61 3 83872305; Fax: +61 3 93874030. Email: dames@unimelb.edu.au. Received 15 Apr 2011; revision requested 5 May 2011; revised version received 27 May 2011; accepted 31 May 2011. First published online 13 July 2011. present to $2.8 \%$ by 2050 (Access Economics, 2009). As is the case elsewhere, Alzheimer's disease $(\mathrm{AD})$ is the main cause of dementia in Australia. $\mathrm{AD}$ is a progressive disorder, but the rate of cognitive decline in $\mathrm{AD}$ varies considerably between individuals, with some patients showing rapid and substantial cognitive decline in a relatively short time and others showing little or no change 
over years (Doody et al., 2001; Capitani et al., 2004). Patients with rapid cognitive decline have a worse prognosis in terms of function and mortality (O'Hara et al., 2002; Holtzer et al., 2003). A number of studies (Sona et al., submitted) have tried to find factors associated with rapid cognitive decline (usually defined in terms of decline in MMSE score), because the prior identification of patients likely to decline quickly could be useful for care planning and might one day permit the application of early interventions designed to slow decline or reduce carer burden.

The Australian Imaging, Biomarkers and Lifestyle (AIBL) study of aging (Ellis et al., 2009) is a large longitudinal study designed to enhance knowledge about AD. It is a twocenter (Melbourne and Perth), prospective study using neuroimaging, biomarkers, clinical and neuropsychological measures, and diet and lifestyle patterns in a cohort of 1112 volunteers comprising patients with AD or mild cognitive impairment (MCI; Winblad et al., 2004; Petersen et al., 2009) and healthy volunteers. AIBL was launched in November 2006 and comprehensive baseline data collection and follow-up assessments (18 months post-baseline) have been completed. It is expected that 3-year follow-up data will be available for analysis within 12 months and collection of data at 54 months will commence during 2011. The baseline cohort included 211 patients (180 probable and 31 possible) with $\mathrm{AD}$ diagnosed according to the NINCDS-ADRDA criteria (McKhann et al., 1984; when the study commenced these were the most up to date clinical research diagnostic criteria for AD available). It is this cohort which is the specific focus of this paper. The aim of this study was to identify factors associated with, or predictive of, rapid cognitive decline in these $211 \mathrm{AD}$ patients.

\section{Methods}

The population at baseline and methods of the AIBL study are fully described in an earlier paper (Ellis et al., 2009). Participants in the AIBL study were invited to undergo reassessment 18 months after a baseline assessment. At both time points data collection included comprehensive cognitive testing, drawing of $80 \mathrm{ml}$ of blood, and completion of health and lifestyle questionnaires. One quarter of the subjects underwent cerebral PET imaging with Pittsburgh Compound B (PiB) (Rowe et al., 2010). Assessments took place at three locations in Melbourne and at two locations in Perth, depending on whether the participants were to undergo brain imaging and where they lived. A small number of participants with advanced $\mathrm{AD}$ were assessed by AIBL staff where they lived. All assessments were conducted in the mornings, after an overnight fast. Weight, height, abdominal girth, sitting blood pressure and pulse were measured, followed by the drawing of $80 \mathrm{ml}$ of blood. Participants were then provided with breakfast, followed by cognitive and mood assessments, including the Mini-Mental State Examination (MMSE; Folstein et al., 1975). For a comprehensive account of the cognitive battery and the rationale behind the selection of individual items, see our baseline paper (Ellis et al., 2009). On completion of the 18-month follow-up assessments, a database of the $211 \mathrm{AD}$ patients was created and analysis was performed on this subgroup only. For the definition of rapid cognitive decline we used a drop of 6 points or more on the MMSE between the baseline and the 18-month follow-up. The threshold of 6 points was selected on the basis of the literature, which suggests a loss of 3 points per year as the mean rate of decline on the MMSE in patients with AD (Clark et al., 1999). Analysis was also conducted with a threshold of 4, 5, 7 and 8 points. Another set of analyses was conducted on deceased patients $(n=$ 17) and on patients with " $\mathrm{AD}$ too severe for followup" ( $\mathrm{n}=9)$ as rapid cognitive decliners. Last, we also conducted analyses excluding the 31 patients classified as "possible AD" by NINCDS-ADRDA criteria.

We searched for a correlation between rapid cognitive decline and those variables which were collected from participants during their baseline AIBL visit, and which have been assessed in other studies of rapid cognitive decline in AD: age, sex, family history of dementia, level of education, smoking habits, diabetes, hypertension, angina or heart attack, cholesterol levels, C-Reactive Protein (CRP), cerebrovascular disease, ApoE genotype, brain derived neurotrophic factor (BDNF), treatment with cholinesterase inhibitors (CheI) and baseline cognitive function as assessed by the Clinical Dementia Rating scale (CDR; Morris, 1993) and the MMSE (Folstein et al., 1975) scores (for a detailed review see Sona et al., submitted).

\section{Statistical analysis}

All the analyses used the statistical package " $R$ " ( $\mathrm{R}$ Development Core Team, 2010). In all the analyses "family history", "smoker", "smokes more than one packet a day", "hypertension", "angina", "heart attack", "ApoE", "CheI" were considered as categorical variables with values "yes" or "no". "Education" was categorized as over 12 years or not more than 12 years, and sex has "female" or "male" values. Other variables including "age", "CDR score", "CDR sum of Boxes", “cholesterol", "triglycerides", "HDL 
cholesterol", "LDL cholesterol", "BDNF", "CRP" and "MMSE" - were treated as continuous variables, as they were recorded.

In univariate analyses, an odds ratio was calculated for each categorical variable comparing the rapid cognitive declining group with the rest. A 95\% confidence level was chosen for calculating the confidence interval. The comparisons of each continuous variable between the two groups were performed with a t-test, and a $\mathrm{p}$ value of 0.05 (one tail) was used to define statistical significance. For multivariate analysis, a multivariate logistic regression model was constructed based on all variables mentioned in this study.

\section{Results}

Of the $211 \mathrm{AD}$ patients recruited at baseline, we had available data for $156(73.9 \%)$ patients at 18 months. The reasons why some patients did not undergo 18-month reassessment are shown in Figure 1. Characteristics of the $\mathrm{AD}$ population at baseline and follow-up are shown in Table 1. The mean baseline MMSE for 211 subjects was 19.0 $( \pm 5.2)(19.1 \pm 5.2$ at baseline for the 156 patients with data at both time points), while at 18 -month follow-up it was $15.4( \pm 7.0)$ for 156 subjects, with a mean drop in the score of $3.8( \pm 4.5)$ points. The mean baseline CDR "sum of boxes" for 211 subjects was $5.7( \pm 2.9)$, while at 18 -month follow-up for 156 subjects with data it was $8.4( \pm 4.3)$ with a mean gain of $3.0( \pm 3.0)$. One-hundred-and-twenty-eight patients $(60.7 \%)$ were prescribed a CheI at baseline and 12 of these patients were taking concomitant memantine. No patient was prescribed memantine without a CheI.

Compared to the 156 patients who could be reassessed after 18 months, patients who did not return for follow-up $(\mathrm{n}=55)$ were older (mean age 81.7 vs 76.9 years, $p=0.001$ ), had higher baseline CDR scores ( 1.2 vs $0.9, \mathrm{p}=0.024)$ and CDR sum of boxes scores (6.7 vs $5.4, \mathrm{p}=0.016)$, and had slightly lower baseline MMSE scores (18.4 vs 19.2, $\mathrm{p}=0.342)$. There were no significant differences in sex ratios or years of education for those who did and did not return for follow-up, but those who did not return for follow-up were significantly more likely not to carry the APOE $\varepsilon 4$ allele $(26 / 55(47 \%)$ APOE $\varepsilon 4$ positive vs $106 / 156(68 \%) \mathrm{p}=0.01$, onetailed). Subjects who could not be reassessed were less likely to have been taking a CheI at baseline than those who returned for follow up $(25 / 55(45 \%)$ taking a CheI vs $103 / 156(66 \%), p=0.005$, onetailed).

Table 2 shows the statistically significant correlations between rapid cognitive decline and

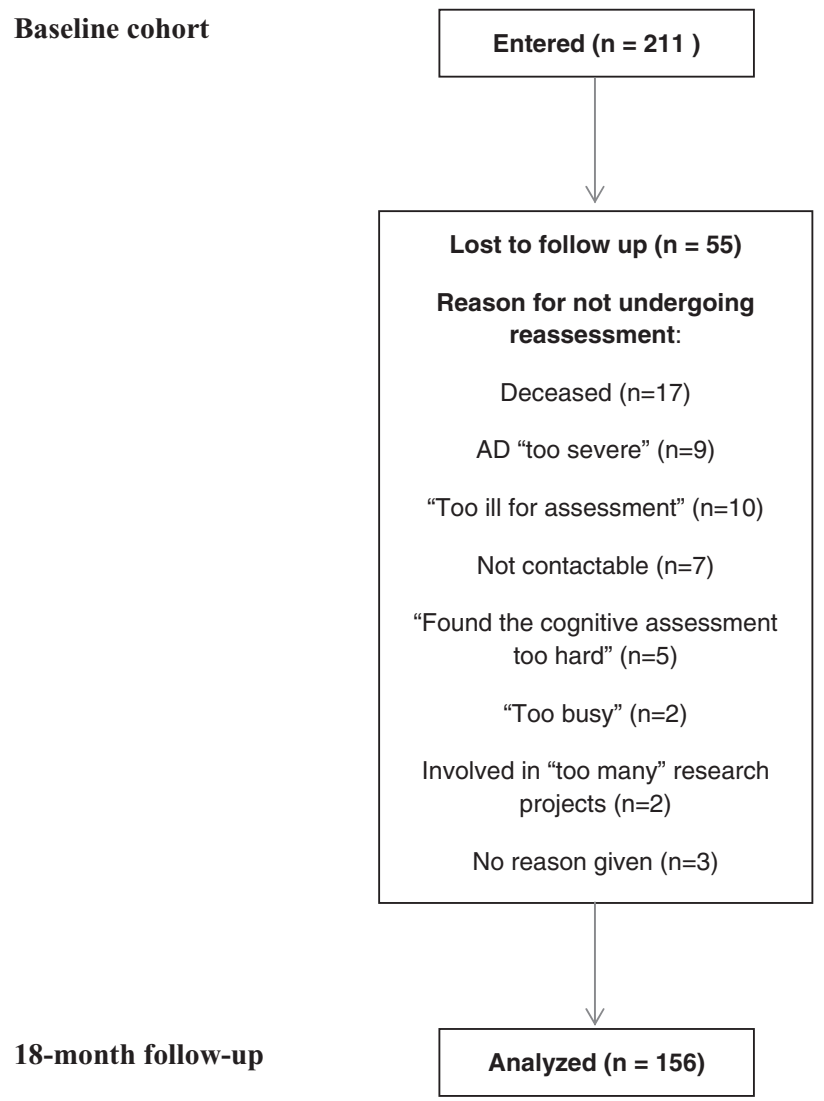

Figure 1. Patient flow

the analyzed factors. Univariate analysis showed that a higher CDR and CDR "sum of boxes" scores and use of a CheI were predictors of rapid cognitive decline in this population. No significant correlation with rapid cognitive decline was found for age, family history of dementia, level of education, presence of any cardiovascular risk factor (smoking habits, diabetes, hypertension, angina or heart attack, cholesterol levels, CRP), cerebrovascular disease, ApoE genotype or BDNF. In the multivariate analysis (logistic regression model) younger age, male sex and CheI treatment made a significant contribution to distinguishing the rapid cognitive decliners from the slow decliners.

The multivariate logistic regression model in this study was used to give the adjusted odds ratios corresponding to the variables in univariate analysis. It simply included all the variables used for the univariate analysis without investigation of interactions between the variables or any variable selection. The odds ratio results from the model showed that there might be some confounding or high correlations between some variables. We investigated the correlations between the variables. CDR score and CDR sum of Boxes are highly correlated as expected (Spearman correlation coefficient 0.8 ). Total cholesterol is also 
Table 1. Characteristics of the sample (baseline and 18-month follow-up)

\begin{tabular}{|c|c|c|}
\hline CHARACTERISTIC & $\begin{array}{l}\text { BASELINE } \\
\operatorname{MEAN}( \pm S D) \text { OR NUMBER( } \%)\end{array}$ & $\begin{array}{l}\text { FOLLOW-UP } \\
\operatorname{MEAN}( \pm S D) \text { OR NUMBER }(\%)\end{array}$ \\
\hline AD patients & $211(100 \%)$ & $156(73.9 \%)$ \\
\hline Age & $78.1( \pm 8.6)$ & $76.9( \pm 8.1)$ \\
\hline Female & $130(61.6 \%)$ & $96(61.5 \%)$ \\
\hline $\operatorname{ApoE} \varepsilon 4+$ & $132(62.6 \%)$ & $106(67.9)$ \\
\hline Years of education ( $\leq 12$ years) & $121(59.1 \%)$ & $85(55.5 \%)$ \\
\hline MMSE & $19.0( \pm 5.2)$ & $15.4( \pm 7.0)$ \\
\hline CDR & $1.0( \pm 0.5)$ & $1.4( \pm 0.8)$ \\
\hline CDR Sum of Boxes & $5.7( \pm 2.9)$ & $8.4( \pm 4.3)$ \\
\hline $\begin{array}{l}\text { Rapid cognitive decliners (loss } \\
\text { of } 6 \text { MMSE points or more) }\end{array}$ & - & $51(32.7 \%)$ \\
\hline
\end{tabular}

$\mathrm{SD}=$ Standard Deviation; AD = Alzheimer's disease; MMSE = Mini-Mental State Examination; CDR = Clinical Dementia Rating scale; CheI = cholinesterase inhibitor.

Table 2. Predictors of rapid cognitive decline (statistically significant results in italics)

\begin{tabular}{|c|c|c|c|c|c|c|c|c|}
\hline \multirow{3}{*}{$\begin{array}{l}\text { CATEGORICAL } \\
\text { VARIABLES }\end{array}$} & \multicolumn{5}{|c|}{$\begin{array}{l}\text { MULTIVARIATE ANALYSIS（LOGISTIC } \\
\text { REGRESSION） }\end{array}$} & \multicolumn{3}{|c|}{ UNIVARIATE ANALYSIS } \\
\hline & LR & & & & & & & \\
\hline & COEFFICIENT & OR & 95 & $\%$ CI & P VALUES & OR & $95 \%$ & CI \\
\hline $\begin{array}{l}\text { Family history of } \\
\text { dementia }\end{array}$ & 0.7 & 2.0 & 0.8 & 5.4 & 0.16 & 1.7 & 0.8 & 3.5 \\
\hline Smoking & 0.1 & 1.2 & 0.4 & 3.4 & 0.79 & 1.1 & 0.5 & 2.4 \\
\hline $\begin{array}{l}\text { Smokes } \geq 1 \text { packet of } \\
\text { cigarettes }\end{array}$ & 1.9 & 6.9 & 1.1 & 49.5 & 0.04 & 2.4 & 0.6 & 9.7 \\
\hline Stroke or TIA & 1.1 & 2.9 & 0.8 & 10.9 & 0.11 & 1.8 & 0.7 & 4.4 \\
\hline Diabetes & 0.1 & 1.1 & 0.2 & 5.7 & 0.92 & 0.9 & 0.3 & 2.6 \\
\hline Hypertension & -0.4 & 0.7 & 0.2 & 1.9 & 0.45 & 0.8 & 0.4 & 1.7 \\
\hline Angina & 0.4 & 1.6 & 0.3 & 8.8 & 0.62 & 0.8 & 0.2 & 2.6 \\
\hline Heart attack & 0.8 & 2.2 & 0.3 & 17.5 & 0.46 & 1.1 & 0.2 & 4.2 \\
\hline $\operatorname{ApoE} \varepsilon 4$ & -0.1 & 0.9 & 0.3 & 3.0 & 0.90 & 1.2 & 0.6 & 2.8 \\
\hline Education ( $>12$ years) & 0.2 & 1.2 & 0.4 & 3.5 & 0.73 & 0.9 & 0.4 & 1.9 \\
\hline CheI & 1.5 & 4.3 & 1.3 & 17.6 & 0.03 & 3.4 & 1.5 & 8.9 \\
\hline Gender (male) & -1.3 & 0.3 & 0.1 & 0.9 & 0.05 & 0.6 & 0.3 & 1.3 \\
\hline \multicolumn{9}{|l|}{ CONTINUOUS } \\
\hline VARIABLES & & & & & & RCD Mean & SCDMean & $\mathrm{P}$ values \\
\hline Age & -0.1 & 0.9 & 0.9 & 1.0 & 0.05 & 76.7 & 77.0 & 0.9 \\
\hline CDR score & 1.4 & 4.2 & 0.4 & 48.3 & 0.23 & 1.1 & 0.9 & 0.0 \\
\hline CDR sum of boxes & 0.1 & 1.1 & 0.7 & 1.7 & 0.73 & 6.3 & 5.1 & 0.0 \\
\hline Total cholesterol & 2.8 & 16.3 & 0.0 & $>1000$ & 0.76 & 5.3 & 5.5 & 0.4 \\
\hline Triglycerides & -1.1 & 0.3 & 0.0 & $>1000$ & 0.80 & 1.3 & 1.4 & 0.5 \\
\hline HDL cholesterol & -2.7 & 0.1 & 0.0 & $>1000$ & 0.77 & 1.7 & 1.7 & 0.5 \\
\hline LDL cholesterol & -3.1 & 0.0 & 0.0 & $>1000$ & 0.74 & 3.0 & 3.1 & 0.3 \\
\hline BDNF & -0.1 & 0.9 & 0.8 & 1.1 & 0.53 & 1.8 & 1.8 & 0.9 \\
\hline CRP & -0.1 & 0.9 & 0.8 & 1.0 & 0.37 & 2.3 & 3.3 & 0.3 \\
\hline MMSE & -0.02 & 1.0 & 0.8 & 1.1 & 0.75 & 18.0 & 19.6 & 0.1 \\
\hline
\end{tabular}


highly correlated with LDL cholesterol (Pearson correlation coefficient 0.9 ).

Analysis with a threshold of $4,5,7$ or 8 MMSE points to define rapid decline showed no trend towards significance for any other variables but confirmed the same correlations with baseline functional and cognitive status and CheI treatment. Analysis was also carried out on the deceased patients $(n=17)$ and the patients whose $A D$ was "too severe" $(\mathrm{n}=9)$ as rapid cognitive decliners and this statistical analysis showed no significant difference from previous ones regarding factors associated with rapid cognitive decline. Excluding the 31 "possible $\mathrm{AD}$ " subjects from the analyses did not affect any of the results.

\section{Discussion}

The first issue raised by this work is the definition of rapid cognitive decline (for a detailed review of this subject see Sona et al., submitted). A recent consensus paper (Soto et al., 2008a) sought to establish a definition of rapid cognitive decline, with the authors proposing "the loss of 3 points or greater in MMSE during six months" . In their paper the authors noted that only a few studies had tried to define rapid cognitive decline: in an earlier paper, Soto et al. (2005) proposed a 4 point or greater loss on the MMSE within 6 months and the loss of at least 1 more point on the MMSE during the following six months; Carcaillon et al. (2007), in their bid to find a significant threshold of decline associated with a higher mortality rate, proposed a loss of 3 points or greater per year on the MMSE; Soto et al. (2008b) concluded that the loss of 4 points or more in the MMSE during the first 6 months of follow-up was a predictor of a worse clinical course of $\mathrm{AD}$. Two later papers give a definition of rapid cognitive decline based on the MMSE: Doody et al. (2010) who used the same definition as in a previous study (Doody et al., 2001), dividing patients into slow progressors (decline of less than 2 points per year on the MMSE), intermediate progressors ( 2 to 4 points per year) and rapid progressors $(\geq 5$ points per year); and Musicco et al. (2010) who considered the time-dependent probability of losing 5 points on the MMSE over two years as rapid disease progression. The rationale behind our choice of the loss of 6 points or more over 18 months is based on the fact that cognitive decline is not linear, as is demonstrated by recent research (Mendiondo et al., 2000). Also, the literature suggests a loss of some 3 points per year as the mean rate of decline on the MMSE in AD (Clark et al., 1999), so 6 points lost in 18 months clearly would be faster than the average rate of decline. We were constrained in our assessments of decline by the fact that funding only permits reassessment of the AIBL cohort every 18 months. Most of the studies noted above gave a definition of rapid cognitive decline based on the rate of decline of their particular population, but this procedure could enhance intrinsic variation among the studied subjects. We chose to give a reasonable (even if partly empirical) definition of rapid decline which is independent of our population characteristics and easily reproducible. Furthermore, we repeated our analyses using different MMSE decline thresholds, without and with patients who died and those whose symptoms were too severe for follow-up, and both including and excluding NINCDS-ADRDA possible $\mathrm{AD}$ patients, and no significant change in our results was found.

The main findings of this study are: (1) nearly a third of patients satisfying strict clinical criteria for $\mathrm{AD}$ are likely to experience a rapid cognitive decline; (2) worse baseline cognitive function and greater functional impairment are correlated with rapid cognitive decline; and (3) treatment with a CheI at baseline is also associated with a higher risk of rapid cognitive decline.

Using a definition of rapid cognitive decline as a drop of 6 points on the MMSE, almost one third of the patients had declined to this extent after 18 months. This figure is consistent with other reports: for example, Atchison et al. (2004) and Carcallion et al. (2007) reported $33.6 \%$ and $33.9 \%$ of patients respectively who were rapid cognitive decliners. This subgroup of patients may need a higher level of attention than their counterparts. As they are declining rapidly, one could argue that they should be the target of specific measures to delay their cognitive deterioration. Unfortunately, the lack of clearly defined characteristics associated with rapid cognitive decline (Sona et al., submitted) makes the early recognition of these patients extremely difficult, and to date there is a lack of effective disease modifying strategies available for AD.

The finding of an association between initial cognitive and functional status (as defined by the CDR scale) and subsequent decline is not surprising, as many studies demonstrate that patients who are more severely impaired in the early stages are more likely to decline faster than less impaired patients (Morris et al., 1993; Doody et al., 2001; Bhargava et al., 2006; Ousset et al., 2008). But the literature also contains some contradictory results: Burns et al. (1991) found that cognitive function at baseline was associated with accelerated decline only for patients at an intermediate level of impairment at baseline; and a very recent paper published in this journal (Brodaty 
et al., 2011) demonstrated in a large sample of patients $(\mathrm{n}=967)$ attending several different memory clinics in Australia that higher baseline MMSE scores (better cognition) were associated with faster cognitive decline over a period of 6 months. The authors pointed out that 6 months is a relatively short period of time to detect predictors of disease progression and that a floor effect could not be ruled out as an influencing factor, since the most impaired patients (those at the low end of MMSE score) had less room to decline over the 6-month period. Different recruitment settings (memory clinics versus volunteers) could go some way towards explaining these differences, as those who volunteer for research studies are likely to form a selected subsample of those with $\mathrm{AD}$ diagnosed via memory clinics. Furthermore, these results appear to be consistent with the course of decline in MMSE scores from early to severe stages of $\mathrm{AD}$ : subjects with intermediate stages of disease tend to have a greater decline in cognitive function than those with mild or severe impairment. Graphically, the course of cognitive decline assessed by the MMSE scale would look like a sinusoid, suggesting that the scale is more sensitive to changes in the middle to late stages of $\mathrm{AD}$ (Mendiondo et al., 2000). Also, cognitive tests may not be sensitive to all stages of the disease, especially for patients with the least and the greatest impairments (Flicker, 2010).

Our finding of a higher risk of being a rapid cognitive decliner when treated with a CheI was surprising to us, given the internationally recognized beneficial effect of CheIs in the mild to moderate stages of $\mathrm{AD}$, which has been documented in numerous prospective randomized controlled trials (Birks, 2006). In addition, at least one other study found that the use of CheIs for at least one year was associated with a decreased risk of rapid cognitive deterioration (Gillette-Guyonnet et al., 2006). However, our surprise was attenuated when we read the recent paper of Schneider et al. (2011), which reports more rapid decline in people with both MCI and AD in the ADNI cohort who were prescribed either a CheI or memantine (we were unable to analyze the effect of memantine on our cohort as only 12 subjects took it at baseline and all were taking a concomitant $\mathrm{ChEI}$ ). As has been suggested in relation to MCI subjects by Schneider et al., perhaps clinicians treating $\mathrm{AD}$ participants in the AIBL study are selecting individuals with more aggressive disease progression for drug therapy, while adopting a more conservative approach to those with slower disease progression. We doubt that differential dropout of slowly progressing patients produced this result, as our dropouts tended to be more impaired at baseline than those we were able to reassess. However, significantly fewer dropouts were prescribed a CheI at baseline than were the 156 subjects who were reassessed, and this may mean that some rapidly declining subjects who were not on a CheI were missed at follow-up, potentially inflating the effect of CheI prescription on the result for those who were reassessed, though it is notable that including the cases who had died or whose $\mathrm{AD}$ was too severe for reassessment in the analysis did not affect the significance of the association with CheI prescription and rapid decline. Of course, it is possible that the finding with respect to baseline CheI prescription occurred by chance, and our results should not be interpreted as a reason to deny established palliative treatment to people affected by $\mathrm{AD}$. We will be interested to see whether any future $\mathrm{AD}$ cohort studies replicate or contradict this result. Our paper relates only to subjects with $\mathrm{AD}$, but future analysis of other AIBL data is unlikely to cast further light on the relationship of CheI prescribing with rapid decline in MCI, as such treatment is rarely used by Australian clinicians for subjects diagnoses with MCI and fewer than 10 AIBL MCI subjects were taking any cognitive enhancer at baseline.

Some limitations need to be acknowledged when reviewing our data. First of all, the number of patients is relatively small (even if very well characterized) and may not represent a sufficiently large sample from which to generalize our results. Second, the outcome choice of a drop of 6 points on the MMSE in 18 months is reasonable, but not universally accepted as a way to define rapid cognitive decline. Last, this study is based on a convenience sample of $\mathrm{AD}$ subjects who volunteered to take part in prospective research and they may not be representative of the wider population of people with $\mathrm{AD}$.

More research is needed to better identify $\mathrm{AD}$ patients who are at higher risk of rapid cognitive decline in order prospectively to assess the effect of interventions (pharmacological and/or social) in delaying progression or reducing costs and to ensure that the best available level of care is provided to such patients.

\section{Conflict of interest declaration}

D. Ames is former Editor-in-Chief of International Psychogeriatrics. He has received payment for advisory board participation and support to attend conferences from companies marketing cognitive enhancing agents.

N. Lautenschlager is the Editor-in-Chief of International Psychogeriatrics. She has received payment for advisory board membership and 
support to attend conferences from companies marketing cognitive enhancing agents.

This paper was peer-reviewed independently through the office of one of the deputy editors.

\section{Description of authors' roles}

A. Sona assembled the data for analysis, assisted with the analysis and wrote drafts of the paper while he was a visiting fellow at NARI from September 2010 to April 2011. P. Zhang designed and executed the statistical analyses and wrote sections of the paper. D. Ames and K. Ellis oversaw the AIBL study in all its aspects, conceived the research question, supervised A. Sona and reviewed and revised successive drafts of the paper. N. Lautenschlager assisted with development of the research question, supervision of the study, confirmation of diagnoses and writing of the paper. A. Bush, R. Martins, C. Masters, C. Rowe and C. Szoeke oversaw the AIBL study and contributed to drafts of the paper. K. Taddei managed AIBL data collection in Perth and contributed to drafts of the paper.

\section{Acknowledgments}

Core funding for the study was provided by CSIRO, which was supplemented by "in kind" contributions from the study partners (Australian Commonwealth Scientific Industrial and Research Organization (CSIRO), University of Melbourne, Neurosciences Australia Ltd. (NSA), Edith Cowan University (ECU), Mental Health Research institute (MHRI), Alzheimer's Australia (AA), National Ageing Research Institute (NARI), Austin Health, University of WA (UWA), CogState Ltd., Macquarie University, Hollywood Private Hospital, and Sir Charles Gairdner Hospital). The AIBL investigators thank Richard Head of CSIRO for initiating and facilitating the AIBL collaboration. The study also received support from the National Health and Medical Research Council (NHMRC) via the Dementia Collaborative Research Centres program (DCRC2). Pfizer International has contributed financial support to assist with analysis of blood samples and to further the AIBL research program. Cassandra Szoeke has been partially supported by research fellowships funded by Alzheimer's Australia and the NHMRC. Alzheimer's Australia (Victoria and Western Australia) assisted with promotion of the study and the screening of telephone calls from volunteers. The AIBL team wishes to thank the clinicians who referred patients with $\mathrm{AD}$ to the study: Professor David Ames, Associate
Professor Brian Chambers, Professor Edmond Chiu, Dr Roger Clarnette, Associate Professor David Darby, Dr Mary Davison, Dr John Drago, Dr Peter Drysdale, Dr Jacqueline Gilbert, Dr Kwang Lim, Professor Nicola Lautenschlager, Dr Dina LoGiudice, Dr Peter McCardle, Dr Steve McFarlane, Dr Alastair Mander, Dr John Merory, Professor Daniel O'Connor, Professor Christopher Rowe, Dr Ron Scholes, Dr Mathew Samuel, Dr Darshan Trivedi, and Associate Professor Michael Woodward. We thank all those who participated in the study for their commitment and dedication to helping advance research into the early detection and causation of $\mathrm{AD}$.

\section{References}

Access Economics (2009). Keeping Dementia Front of Mind: Incidence and Prevalence 2009-2050. Canberra: Alzheimer's Australia.

Atchison, T. B., Bradshaw, M. and Massman, P. J. (2007). Investigation of profile difference between Alzheimer's disease patients declining at different rates: examination of baseline neuropsychological data. Archives of Clinical Neuropsychology, 19, 1007-1015.

Birks, J. (2006). Cholinesterase inhibitors for Alzheimer's disease. Cochrane Database of Systematic Reviews, 1, CD005593.

Bhargava, D., Weiner, M. F., Hynan, L. S., Diaz-Arrastia, R. and Lipton, A. M. (2006). Vascular disease and risk factors, rate of progression, and survival in Alzheimer's disease. Fournal of Geriatric Psychiatry and Neurology, 19, 78-82.

Brodaty, H., Woodward, M., Boundy, K., Ames, D. and Balshaw, R. (2011). Patients in Australian memory clinics: baseline characteristics and predictors of decline at 6 months. International Psychogeriatrics. Epublished ahead of print, doi:10.107/S1041610211000688.

Burns, A., Jacoby, R. and Levy, R. (1991). Progression of cognitive impairment in Alzheimer's disease. Fournal of the American Geriatrics Society, 39, 39-45.

Capitani, E., Cazzaniga, R., Francescani, A. and Spinnler, H. (2004). Cognitive deterioration in Alzheimer's disease: is the early course predictive of the later stages? Neurological Sciences, 25, 198-204.

Carcallion, L., Peres, K, Pere, J. J., Helmer, C., Orgogozo, J. M., Dartigues, J. F. (2007). Fast cognitive decline at the time of dementia diagnosis: a major prognostic factor for survival in the community. Dementia and Geriatric Cognitive Disorders, 24, 138-145.

Clark, C. M. et al. (1999). Variability in annual Mini-Mental State Examination score in patients with probable Alzheimer disease. Archives of Neurology, 56, 857862 .

Doody, R. S., Massman, P. and Dunn, K. (2001). A method for estimating progression rates in Alzheimer's disease. Archives of Neurology, 58, 449-454.

Doody, R. S., Pavlik, V., Massman, P., Rountree, S., Darby, E. and Chan, W. (2010). Predicting progression 
of Alzheimer's disease. Alzheimer's Research and Therapy, 2, 2-9.

Ellis, K. A., et al. (2009). The Australian Imaging, Biomarkers and Lifestyle (AIBL) study of aging: methodology and baseline characteristics of 1112 individuals recruited for a longitudinal study of Alzheimer's disease. International Psychogeriatrics, 21, 672-687.

Flicker, L. (2010). Screening and assessment instruments for the detection and measurement of cognitive impairment. In D. Ames, A. Burns, A. and J. O'Brien (eds.), Dementia 4th edn. (p. 57). London: Hodder Arnold.

Folstein, M. F., Folstein, S. E. and McHugh, P. R. (1975). "Mini-mental state": a practical method for grading the cognitive state of patients for the clinician. Fournal of Psychiatric Research, 12, 189-198.

Gillette-Guyonnette, S., et al. (2006). Outcome of Alzheimer's disease: potential impact of cholinesterase inhibitors. Fournals of Gerontology, Series A: Biological Sciences and Medical Sciences, 61, 516-520.

Holtzer, R. et al. (2003). The rate of cognitive decline and risk of reaching clinical milestones in Alzheimer's disease. Archives of Neurology, 60, 1137-1142.

McKhann, G., Drachman, D., Folstein, M., Katzman, R., Price, D. and Stadlan, E. M. (1984). Clinical diagnosis of Alzheimer's disease: report of the NINCDS-ADRDA Work Group under the auspices of the Department of Health and Human Services Task Force on Alzheimer's disease. Neurology, 34, 939-944.

Mendiondo, M. S., Ashford, J. W., Kryscio, R. J. and Schmitt, F. A. (2000) Modeling Mini Mental State Examination changes in Alzheimer's disease. Statistical Medicine, 19, 1607-1616.

Morris, J. C. (1993). The Clinical Dementia Rating (CDR): current version and scoring rules. Neurology, 43, 2412-2414.

Morris, J. C., et al. (1993). The consortium to establish a registry for Alzheimer's disease (CERAD). Part IV. Rates of cognitive change in the longitudinal assessment of probable Alzheimer's disease. Neurology, 43, 2457-2465.

Musicco, M. et al. (2010). Neuropsychological predictors of rapidly progressing patients with Alzheimer's disease.
Dementia and Geriatric Cognitive Disorders, 30, 219228

O'Hara, R. et al. (2002). Which Alzheimer patients are at risk for rapid cognitive decline? Fournal of Geriatric Psychiatry and Neurology, 15, 233-238.

Ousset, P. J., Nourhashemi, F., Reynish, E. and Vellas, B. (2008). Nutritional status is associated with disease progression in very mild Alzheimer disease. Alzheimer Disease and Associated Disorders, 22, 66-71.

Petersen, R. C. et al. (2009). Mild cognitive impairment: ten years later. Archives of Neurology, 66, 1447-1455.

R Development Core Team (2010). R: a Language and Environment for Statistical Computing. Vienna: R Foundation for Statistical Computing. See http://www.R-project.org.

Rowe, C. et al. (2010). Amyloid imaging results from the Australian Imaging, Biomarkers and Lifestyle (AIBL) study of aging. Neurobiology of Aging, 31, 1275-1283.

Schneider, L. S., Insel, P. S. and Weiner, M. W. for the Alzheimer's Disease Neuroimaging Initiative. (2011). Treatment with cholinesterase inhibitors and memantine of patients in the Alzheimer's Disease Neuroimaging Initiative. Archives of Neurology, 68, 58-66.

Sona, A., Ellis, K. and Ames, D. (submitted). Rapid cognitive decline in Alzheimer's disease: a selective review of the literature. Submitted to International Psychogeriatrics.

Soto, M. E., Gillette-Guyonnet, S., Vellas, B. and the REAL.FR group. (2005). Rapid cognitive decline: searching for a definition and predictive factor among elderly with Alzheimer's disease. Fournal of Nutrition, Health and Aging, 9, 158-161.

Soto, M. E. et al. (2008a). Rapid cognitive decline in Alzheimer disease. Consensus paper fournal of Nutrition Health and Aging, 12, 703-713.

Soto, M. E. et al. (2008b). Predictive value of rapid decline in Mini Mental State Examination in clinical practice for prognosis in Alzheimer's disease. Dementia and Geriatric Cognitive Disorders, 26, 109-116.

Winblad, B. et al. (2004). Mild cognitive impairment beyond controversies, towards a consensus: report of the International Working Group on Mild Cognitive Impairment. Fournal of Internal Medicine, 256, 240-246. 